\title{
Analysis of the CMS visitors feedback
}

\section{Elsa Fabiola Vazquez Valencia* ${ }^{*}$}

Universidad Iberoamericana, Ciudad de Mexico.

E-mail: elsa.fabiola.vazquez.valenciaecern.ch

\section{Salvador Carrillo}

Universidad Iberoamericana, Ciudad de Mexico.

E-mail: salvador.carrillo@cern.ch

CMS welcomed over 5500 visitors underground during the 2013 CERN Open Days and more than 4500 during the Neighbourhood Days of 2014 on the occasion of CERN's 60th anniversary. During the latter event, visitors gave their feedback on the visit experience by answering three questions:

- In one sentence, what will you tell your friends about what you saw today?

- What fact or story that you heard today impressed you the most?

- Describe the CMS detector in three words.

This poster will show the analysis of the answers given by visitors.

The European Physical Society Conference on High Energy Physics 22-29 July 2015

Vienna, Austria

${ }^{*}$ Speaker.

$\dagger_{\text {joint }}$ effort with the CMS Communications Group 


\section{Introduction}

CMS welcomed over 5500 visitors underground during the 2013 CERN Open Days and more than 4500 during the Neighbourhood Days of 2014 on the occasion of CERN's 60th anniversary.

During the Open Days (28 and 29 September, 2011) 43 activities were organized across all the CERN sites: visits to laboratories, workshops, accelerators and detectors, demonstrations of CERN's technologies, research-based lectures and presentations, etc. The entrance was free for all members of the public. [1]

To celebrate its 60th anniversary, CERN had invited residents of the local France-Geneva region to come and celebrate at a special event held on 24 and 25 May, 2014. 8000 of them accepted the invitation to visit installations that many of them pass by every day. Guided tours were offered at three of the Laboratory's underground sites: the CMS and LHCb detectors and the LHC accelerator at Point 4 of the ring. The lifts went up and down non-stop for the whole weekend, taking enthusiastic visitors underground to get behind the scenes of fundamental research. The caverns and the tunnel echoed to the oohs and aahs of people enthralled by the size and complexity of the installations so close to their homes. [2]

At the CMS site in Cessy, a whole host of activities had also been organized above ground. Young and not-so-young visitors had fun shooting virtual protons, using Lego to get to grips with the components of matter, building complex machines out of Kapla blocks, testing their dexterity with robots or taking the helm of a huge crane that's more commonly used to move the heavier components of CERN's installations. Students from Cessy school, a neighbour of the CMS experiment, challenged visitors with the mysterious boxes that form part of the" Be a Physicist " project that they run in cooperation with CERN. Stands devoted to radiation protection and superconductivity, cryogenics shows and precision measurement demonstrations all drew big crowds.

Some of the visitors left messages before leaving, including this one:" What struck me the most was the idea that so many people from all around the world are working together towards a better understanding of the Universe."

Our poster is based on these messages.

\section{Analysis}

During the Neighbourhood Days of 2014, on the occasion of CERN's 60th anniversary, people were asked to gave their feedback on the visit experience by answering three questions:

- In one sentence, what will you tell your friends about what you saw today?

- What fact or story that you heard today impressed you the most?

- Describe the CMS detector in three words. 
In total we analyzed 495 bilingual questionnaires, where the three questions were stated. 234 questionnaires were answered in french, and 215, in english.

We made a database with all the key words that were used to answer each question. The key words are adjectives, adverbs, verbs or nouns. We count the words, no matter if it was in english or french. In total we count about 235 different words for the first question, 270 different words for the second question, and 190 different words for the third question.

In order to produce some graphs we only took into account words that appeared more than 15 , 14 or 13 times. The results for the " most used words" are in the following histograms:

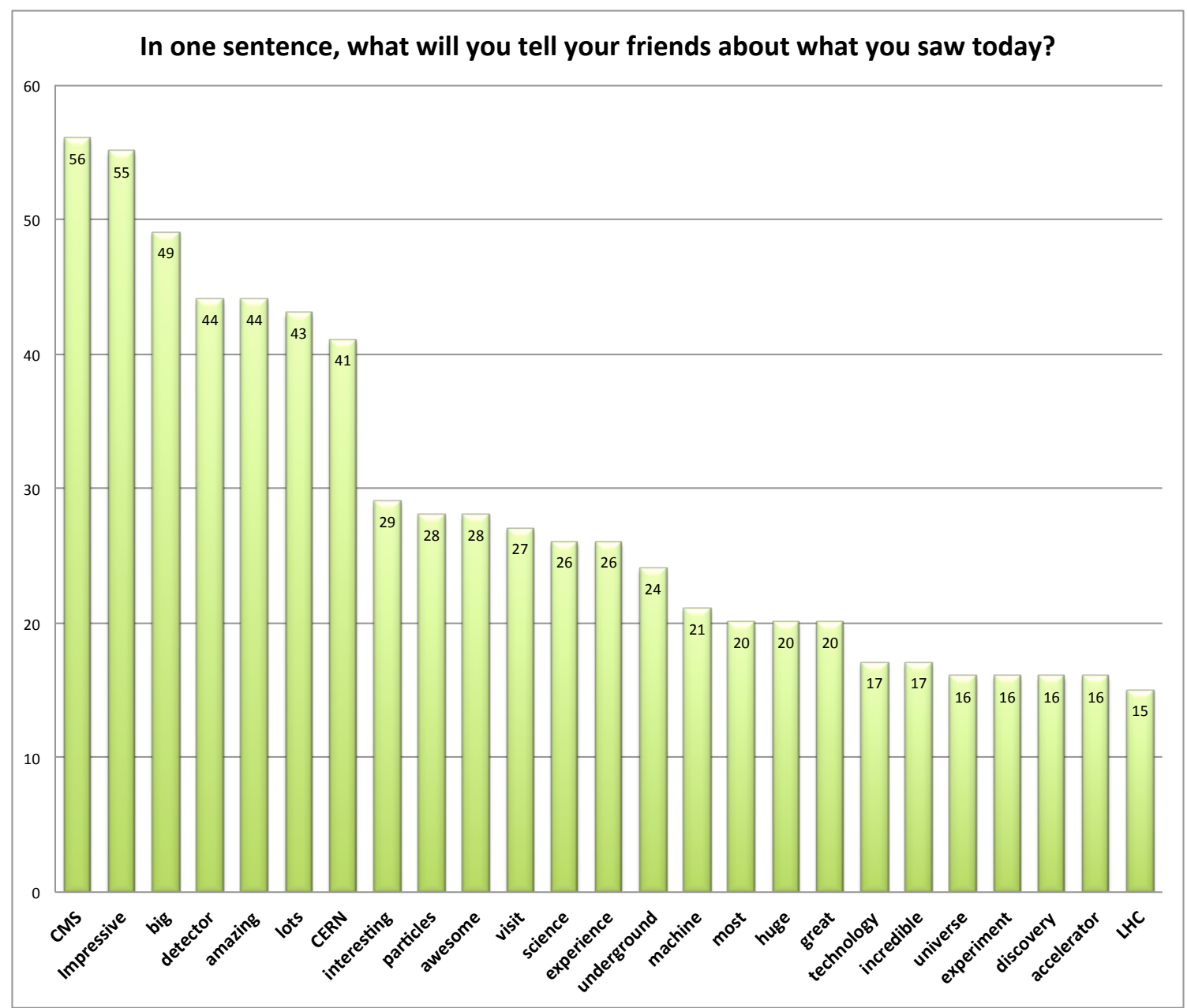

Figure 1: Most used words for question 1. 


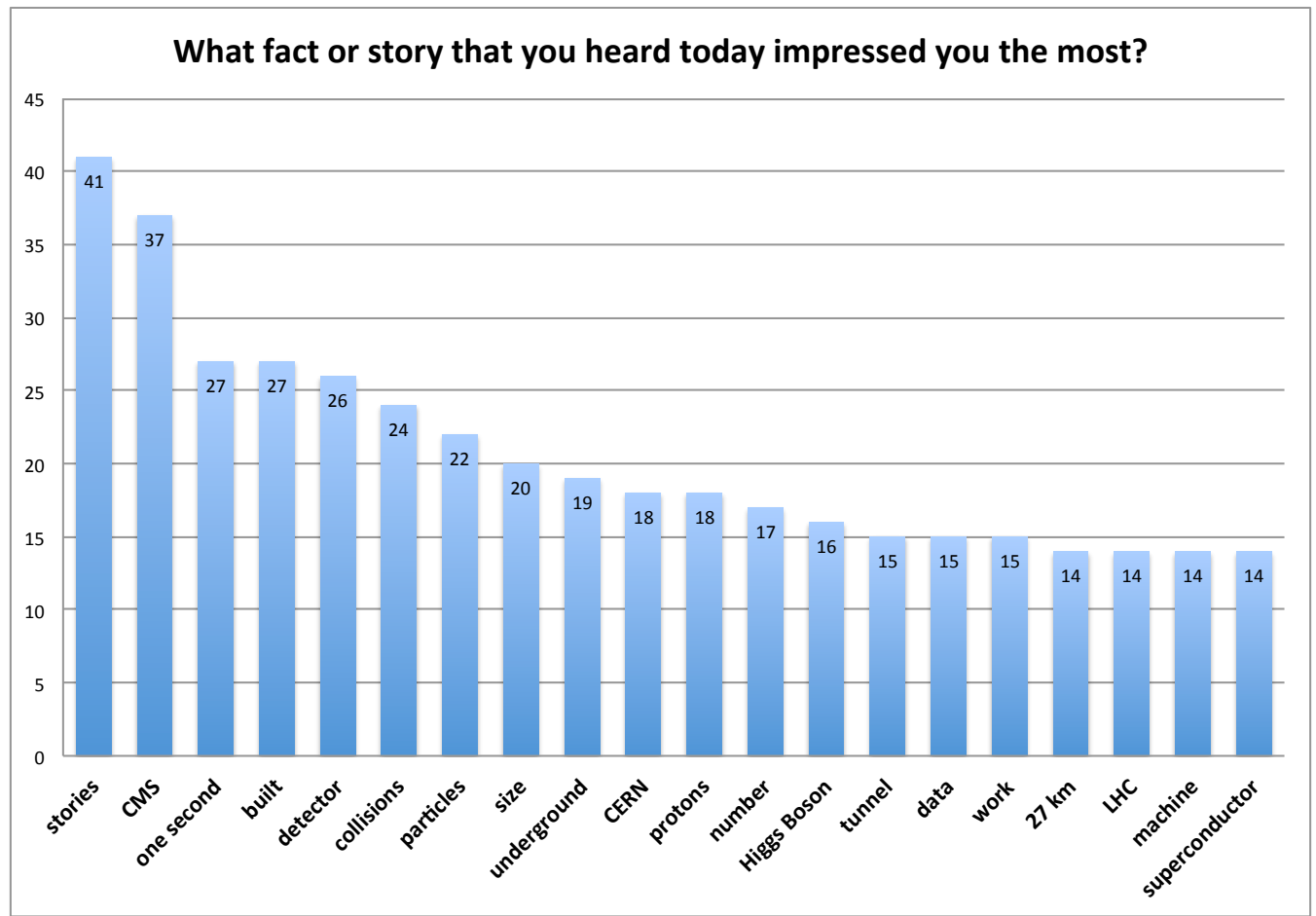

Figure 2: Most used words for question 2.
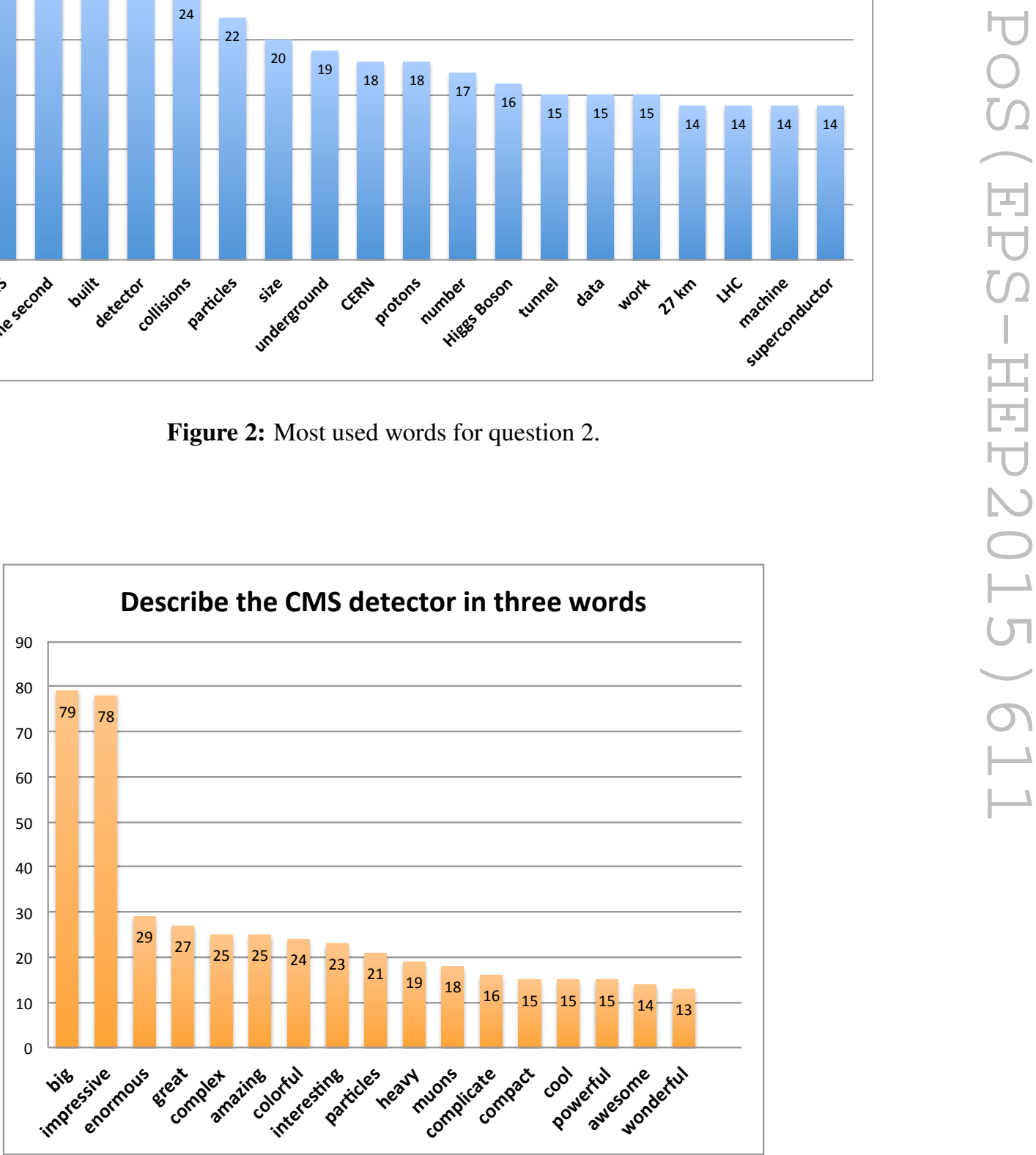

Figure 3: Most used words for question 3. 


\section{Conclusion}

In the poster, instead of only put the data like this, we wanted to present them in a more artistic way. We used the words to form some iconic pictures. Also we presented the words in boards where the size of the word is proportional to the times it appears.

This is how the idea of the poster was developed.

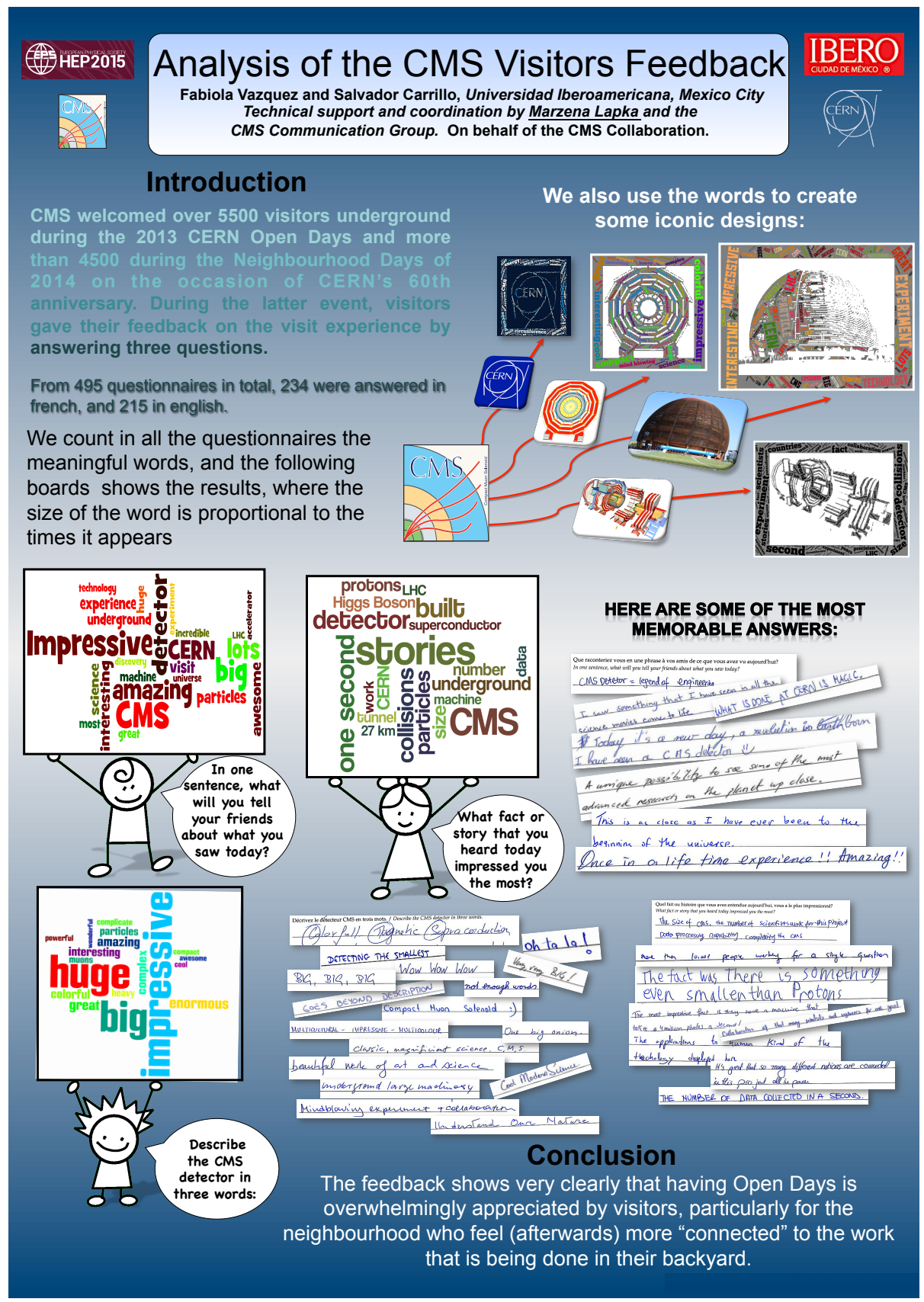

Figure 4: Poster 


\section{References}

[1] "CERN Accelerating Science." CERN Opens Its Doors September 28th. N.p., n.d. Web. 23 Oct. 2015.

[2] "CERN Accelerating Science." CERN Celebrates Its Anniversary with Its Neighbours. N.p., n.d. Web. 23 Oct. 2015. 